\title{
Migrating Planets
}

\author{
N. Murray ${ }^{1}$, B. Hansen ${ }^{1}$, M. Holman ${ }^{1}$, and S. Tremaine ${ }^{1,2}$ \\ ${ }^{1}$ Canadian Institute for Theoretical Astrophysics, \\ University of Toronto, Toronto, Ontario M5S 3H8, Canada \\ ${ }^{2}$ Canadian Institute for Advanced Research, \\ Program in Cosmology and Gravity
}

Received __; accepted _ 


\begin{abstract}
A planet orbiting in a disk of planetesimals can experience an instability in which it migrates to smaller orbital radii. Resonant interactions between the planet and planetesimals remove angular momentum from the planetesimals, increasing their eccentricities. Subsequently, the planetesimals either collide with or are ejected by the planet, reducing the semimajor axis of the planet. If the surface density of planetesimals exceeds a critical value, corresponding to $\sim 0.03$ solar masses of gas inside the orbit of Jupiter, the planet will migrate inward a large distance. This instability may explain the presence of Jupiter-mass objects in small orbits around nearby stars.
\end{abstract}

In the standard theory of solar system formation, solid material orbiting in a gaseous disk accumulates to form small rocky or icy bodies called planetesimals ([1). Protoplanets then form by accretion of planetesimals. If a protoplanet accretes roughly ten Earth masses $\left(10 M_{\oplus}\right)$, it can then capture a gas envelope from the protoplanetary disk and become a gas giant like Jupiter. We now know of a number of Jupiter-mass objects orbiting solar-type stars well inside the radius where rocky material can condense (Table 1; ref. 2-8). For example, a planet orbits $\tau$ Bootis at a distance of 0.0462 astronomical units (AU), where the equilibrium temperature of $1550 \mathrm{~K}$ is higher than the condensation temperature of the most refractory minerals. Although some of these detections are controversial (9), we feel it is likely that most or all are real. It is difficult to understand how to form such planets in place.

Although it is difficult to form planets at such small radii, once in place they can survive (10). Thus it is natural to ask whether giant planets can form at orbital radii of a few AU and then migrate inward. One proposed migration mechanism involves the generation by 
the planet of density waves in the gaseous protoplanetary disk, which cause the planet to spiral inward (10, 11). The movement of the planet might be halted by short-range tidal or magnetic effects from the central star (10); however, short-range stopping mechanisms cannot easily explain the objects in Table 1 with semimajor axes $a_{p} \gtrsim 0.2$ AU.

Another migration scenario involves interactions between two or more Jupiter-mass planets $(19,19)$. If two such planets form with a small enough separation their orbits are unstable, resulting in either a collision or an ejection. The typical outcome is a system with a massive planet in an eccentric orbit at 1-2 AU, similar to $70 \mathrm{Vir}$ or HD 114762. In a tiny fraction of cases the periapsis of one of the planets will come close enough to the star that tidal evolution circularizes the orbit, resulting in a massive planet on a circular orbit inside $0.1 \mathrm{AU}$. As pointed out in (19) this low yield is difficult to reconcile with the observed frequency of such systems, roughly a few percent of nearby stars. A second difficulty is the low eccentricity and relatively large $a_{p}$ of the planet orbiting $\rho$ Corona Borealis, which would require an additional damping mechanism.

In this paper we discuss a migration mechanism in which the planet exchanges energy and angular momentum with the residual planetesimal disk through resonant gravitational interactions, gravitational scattering, and physical collisions. Long-distance migration is triggered (the "migration instability") if the surface density $\Sigma$ of planetesimals exceeds a critical value $\Sigma_{c}$ derived below. The migration halts when $\Sigma$ drops below $\Sigma_{c}$, or when a significant fraction of scattered planetesimals strike the star. Since planetesimals cannot survive inside a few stellar radii $\left(R_{*}\right)$, the migration is certain to halt. If the mass of the planet is less than about three times the mass of Jupiter $\left(M_{J}\right)$, the eccentricity of the planet's orbit is reduced by the migration; if not, the eccentricity may increase.

The orbit of a planetesimal is specified by its semimajor axis $a$, eccentricity $e$, and inclination $i$; the corresponding elements for the planet are $a_{p}, e_{p}$, and $i_{p}$. In our analytic 
work, but not in out numerical work, we assume that the mutual inclination between the orbits of planetesimals and planet is $\lesssim 0.1$ radians. It will also be convenient to use the specific energy $E=-G M_{*} /(2 a)$ and angular momentum $L=\left[G M_{*} a\left(1-e^{2}\right)\right]^{1 / 2}$, where $M_{*}$ is the mass of the central star; we shall not distinguish between the total angular momentum $L$ and its normal component $L \cos i$. We assume that the ratio $\mu=M_{p} / M_{*}$ of planet mass to stellar mass is much less than unity $\left(1 / \mu=M_{\odot} / M_{J} \simeq 1047\right.$ for Jupiter $)$, as is the ratio of planetesimal mass to planet mass.

Planet formation remains poorly understood, but we flank this obstacle by focusing on processes that occur after formation is nearly complete. We shall assume for simplicity that only a single massive planet forms. The newly formed planet is probably surrounded by an annular gap in the planetesimal disk, whose radial extent is $\sim 2.5$ times the Hill radius, $h=\mu^{1 / 3} a_{p}$ (四). However, a planet's gravitational reach can exceed its Hill radius grasp by a larger factor, as we now explain.

Consider a planetesimal with orbital period $T=2 \pi\left(a^{3} / G M_{*}\right)^{1 / 2}$ and $e \ll 1$, perturbed by an exterior planet with period $T_{p}>T, a_{p}=a+\Delta a$ and $e_{p} \sim e$. If the planetesimal is in a mean-motion resonance, so that $(k+q) T \simeq k T_{p}$, where $k$ and $q$ are positive integers, the planet can force chaotic perturbations of the planetesimal orbit. The semimajor axis $a$ is nearly constant, since the planetesimal is in resonance, but the eccentricity $e$ undergoes a random walk, gradually diffusing to larger values. Eventually the planetesimal crosses the planet's orbit, after a time (14)

$$
T_{R} \sim \frac{(2 \Delta a)^{2 q-1}}{\mu} \frac{\left(e_{m} e\right)^{2-\kappa}}{e_{p}^{2(q-\kappa)}},
$$

where $\kappa \in[0, q]$ and $q \gtrsim 2$. This time is given in units in which the gravitational constant $G$, $M_{*}+M_{p}$, and $a_{p}$ are equal to unity, so $T_{p}=2 \pi$. The minimum $e$ at which the planetesimal can enter the Hill sphere of the planet is $e_{m} \simeq\left(a_{p}-h\right) / a-1$ (15). For a Jupiter-mass planet at $a_{p}=5.2 \mathrm{AU}$, and a planetesimal in the $7: 4$ resonance with initial eccentricity $e_{0}=0.05$, 
we find $T_{R} \approx 0.5 \times 10^{5}$ yr $(10)$.

The orbital phase of a planet-crossing planetesimal at times of conjunction with the planet is effectively random because of resonance overlap. Thus close encounters between the two objects are common. The first close encounter generally removes the planetesimal from the resonance, but leaves it in a planet-crossing orbit. A planet-crossing planetesimal undergoes a random walk in both $e$ and $a$ (or in $L$ and $E$ ), but the Jacobi constant $J=E-L$ is roughly constant (17). Scaling arguments suggest that the time to random walk out of the system should generally be between $T_{p} / \mu$ and $T_{p} / \mu^{2}$, corresponding to a range $10^{4}-10^{7}$ yr for Jupiter. This range is consistent with the median lifetime of Jupiter-family comets, $5 \times 10^{5} \mathrm{yr}(18)$, and with the lifetimes found in our numerical simulations. For example, if we start a planetesimal in the 7:4 resonance it evolves at roughly constant $E$ or $a$ while $e$ random walks from 0.05 to $\sim 0.3$ over $\sim 3.5 \times 10^{5} \mathrm{yr}$ (Fig. 1). At this point it suffers a close encounter with Jupiter. It then follows curves of constant $J$ until ejected after about $4 \times 10^{5}$ yr.

Planetesimals may also suffer fates other than ejection:

1. Collision with the star: If the planetesimal random walks to sufficiently small $L$ (large $e$ ), it will be absorbed by the star. This channel can become important once the planet has migrated to $a_{p} \lesssim 5-10 R_{*}$. Planetesimals on resonant orbits with $a \lesssim\left(a_{p}-h\right) / 2$ also tend to collide with the star, because these become highly eccentric before they become planet-crossing.

2. Collision with the planet: A planetesimal may collide with the planet before being ejected. The probability is $1-\exp (-P)$, where

$$
P \simeq \frac{2 M_{*} r_{p}}{M_{p} a_{p}} \simeq 2 \times 10^{-3}\left(\mu^{2} \rho\right)^{-1 / 3}\left(\frac{5.2 \mathrm{AU}}{a_{p}}\right),
$$


where $\rho$ is the mean density of the planet. This result shows that about $20 \%$ of orbitcrossing planetesimals will strike Jupiter but most will be ejected (19).

3. Long-term capture into mean-motion resonances: Planetesimals on planet-crossing orbits can be captured temporarily into resonances; this is the inverse of the process described by equation (11), which now gives the typical residence time in the resonance. The median residence time is generally short compared to the age of the solar system. However, a few planetesimals can be trapped for very long times near stable islands (20). Such trapping has been seen in numerical integrations of planet-crossing orbits (21).

Planetesimals start with negative energy and are ejected with positive energy; thus the ejection process must remove orbital energy from the planet, which moves closer to the star (22). This phenomenon is well-known in the context of our solar system: Fernández and Ip (29) suggested that the ejection of planetesimals caused the orbit of Jupiter to shrink by $0.1-0.2 \mathrm{AU}$. This shrinkage can naturally explain the depletion of the outer asteroid belt (14, 24). If the surface density of planetesimals is above the critical value $\Sigma_{c}$, this process is unstable. To calculate $\Sigma_{c}$, we let the semimajor axis of the planet shrink by $\Delta a_{p}$, reducing its energy by

$$
\Delta E_{p} \sim\left|\frac{G M_{*} M_{p} \Delta a_{p}}{2 a_{p}^{2}}\right| .
$$

Planetesimals newly captured into chaotic resonances will be removed from the system, either by ejection or by consumption by the planet or the star. The mass of planetesimals affected is

$$
\Delta M \simeq 2 \pi p_{c} \alpha a_{p} \Sigma\left(\alpha a_{p}\right)\left|\alpha \Delta a_{p}^{(0)}\right|
$$

where $\alpha<1$ is a measure of the average $a / a_{p}$ of the affected planetesimals (25), and $p_{c} \approx 1$ is 
the capture probability. In disposing of a mass $\Delta M$ of planetesimals the planet loses energy

$$
\Delta E_{\alpha} \sim f\left(a_{p}\right) \frac{G M_{*} \Delta M}{2 \alpha a_{p}},
$$

where $f$ is the fraction of the original planetesimal energy that is taken from the planet's orbit; we expect that $f \approx 1$ for $a_{p} \gg R_{*}$ and becomes negative as $a_{p} \rightarrow R_{*}$, where the planet perturbs most planetesimals into the star.

The migration process is unstable if $\left|\Delta E_{\alpha}\right|>\left|\Delta E_{p}\right|$, which requires that the density exceed the critical density $\Sigma_{c}$ :

$$
\Sigma\left(\alpha a_{p}\right) \gtrsim \Sigma_{c}=\frac{M_{p}}{2 \pi a_{p}^{2} \alpha f\left(a_{p}\right) p_{c}} ;
$$

in other words if the mass in the planetesimal disk interior to the planet is of order $M_{p}$, the planet can migrate nearly to the surface of the star.

The migration halts when either (i) the local surface density of planetesimals falls below the critical value, or (ii) a significant fraction of the planetesimals plunge into the star. The local surface density is sure to fall to zero near the star. Solid bodies cannot condense at radii $\lesssim 7 R_{*}$, and existing planetesimals whose orbits might evolve to smaller radii cannot long survive at distances $\lesssim 2 R_{*}$. The minimum semimajor axis achievable by the migration instability is thus a few to ten stellar radii or $\sim 0.03-0.1$ AU.

We have simulated the evolution of a Jupiter-mass body in a planetesimal disk using the Öpik approximation (20); see Fig. 2. The simulations assume that the surface density in planetesimals varies as $\Sigma(r)=\Sigma_{\odot}(1 \mathrm{AU} / r)^{1.5}$, where $\Sigma_{\odot}$, the surface density at $1 \mathrm{AU}$, is a free parameter. The total mass in the planetesimal disk within radius $r$ is then

$$
M_{D} \simeq 1.4 \times 10^{-3} M_{\odot}\left(\Sigma_{\odot} / 10^{3} \mathrm{~g} \mathrm{~cm}^{-2}\right)(r / \mathrm{AU})^{0.5},
$$

and assuming solar metal abundance $(Z=0.02)$ the disk mass in gas interior to Jupiter's orbit is $0.16 M_{\odot}\left(\Sigma_{\odot} / 10^{3} \mathrm{~g} \mathrm{~cm}^{-2}\right)$. 
The energy transfer from planet to planetesimal is approximated as a succession of close encounters until the planetesimal is ejected, strikes the planet or strikes the star. Summing over many encounters, we derive the average efficiency of energy transfer from the planet to planetesimals in a given resonance. These efficiency factors are then used to calculate the evolution of the planet in a disk of a given initial mass profile. For the case where the planet interacts with the 2:1, 3:2, 2:3 and 1:2 resonances, as well as a broad resonance zone in the immediate vicinity of the planet, the onset of the migration instability is at $\Sigma_{\odot} \approx 200 \mathrm{~g} \mathrm{~cm}^{-2}$ (Fig. 2). Equation (6), evaluated with $a_{p}=5.2 \mathrm{AU}, \alpha=0.7, p_{c}=1$, and $f=1$ gives $\Sigma_{c} \approx 70 \mathrm{~g} \mathrm{~cm}^{-2}$, corresponding to $\Sigma_{\odot} \approx 160 \mathrm{~g} \mathrm{~cm}^{-2}$, in good agreement with our numerical estimate. A much higher density $\Sigma_{\odot} \simeq 8000 \mathrm{~g} \mathrm{~cm}^{-2}$ is required for the instability to persist until the planet reaches $0.03 \mathrm{AU}$.

In some cases a gas disk with the surface density required for the migration instability may be nearly gravitationally unstable. The criterion for local gravitational instability of a thin disk is $Q=c \Omega /(\pi G \Sigma)<1$ where $c$ is the sound speed and $\Omega$ is the angular speed (2X). Replacing $\Sigma$ by $\Sigma_{c}$ from equation (6) we find

$$
Q=1.2\left(\frac{r}{1 \mathrm{AU}}\right)^{1 / 2}\left(\frac{T}{300 \mathrm{~K}}\right)^{1 / 2}\left(\frac{Z}{0.02}\right)\left(\frac{M_{*}}{M_{\odot}}\right)^{1 / 2}\left(\frac{M_{J}}{M_{p}}\right)
$$

where $T$ is the disk temperature.

Ejecting a planetesimal removes energy $\Delta E$ and angular momentum $\Delta L$ from the planet. If $|\Delta E / \Delta L|>1$, then $e_{p}$ decreases, while $e_{p}$ increases if $|\Delta E / \Delta L|<1$. For a planet with $M_{p} \lesssim 3 M_{J},|\Delta E / \Delta L|>1$ because the planetesimal must approach within a few Hill radii of the planet to be ejected, and the Hill sphere does not extend into the region where $|\Delta E / \Delta L|<1$ when $E \approx 0$. In this case the ejection of small planetesimals tends to reduce $e_{p}$ and $a_{p}$. However the planet is immersed in a bath of planetesimals, many of which have substantial masses. Interactions between the planet and these objects will tend to produce equipartition between the energy in radial motion of the planet and the planetesimals. If 
most of the planetesimal mass is in objects of mass near $m_{0}$, the expected equilibrium eccentricity of the planet is $e_{e q} \approx\left(m_{0} / M_{p}\right)^{1 / 2}$. This ranges from $0.06-0.006$ for $m_{0}$ between an Earth mass and a lunar mass.

Seven objects in Table 1 have $a_{p}<1 \mathrm{AU}$, and probably did not form in their present orbits. We suggest that at least four of these seven, 51 Pegasus, 55 Cancri, $\rho$ Corona Borealis, and $\tau$ Bootis, migrated to their present semimajor axes through the process described here. The planet around $v$ Andromeda is puzzling because of its rather high eccentricity, $e_{p}=$ $0.15 \pm 0.04$. Such an eccentricity could arise from an encounter with an object of a few $M_{\oplus}$ or the measured eccentricity could be in error. The last two short-period objects on the list, 70 Virginis with $M=6.6 M_{J}$ and HD 114762 with $M=10 \pm 1 M_{J}$, are sufficiently massive that planetesimals scattered from their Hill sphere can be ejected with $|\Delta L|>|\Delta E|$. If so, their large eccentricities could arise from the migration instability. Their relatively large $a_{p}$ might reflect the difficulty of satisfying the instability criterion (6) at small radii when $M_{p}$ is large.

The planet orbiting 47 Ursae Majoris could have formed at its present location; however, we think it more likely that it migrated inward a few AU and halted because $\Sigma(r)$ dropped below $\Sigma_{c}$. The companion to 16 Cygni B is also likely to have migrated inward; its large $e_{p}$ could be due to interactions with the stellar companion to 16 Cygni B (28).

Equation (7) shows that a planetesimal disk mass of $\sim 6 \times 10^{-4} M_{\odot}$ within the planetary orbit is required to initiate the migration instability for a planet with Jupiter's mass and radius, while a much larger mass of $\sim 2 \times 10^{-2} M_{\odot}$ is required to move the planet to 0.03 AU. Infrared observations of solar-mass $\mathrm{T}$ Tauri stars suggest that disks at the upper end of this mass range are rare, but they do occur (29). These observations measure the total mass in particulate matter at $r<100 \mathrm{AU}$, with results in the range $10^{-5}-10^{-2} M_{*}(39)$. The mass in planetesimals would be similar if the efficiency of conversion of dust particles to 
planetesimals is high, and even higher if the observed disks already hide most of their solid material in planetesimal-sized objects.

There is weak observational evidence for a correlation between the presence of shortperiod planets and high stellar metallicity (31). We note two possible explanations for this correlation: (i) for a fixed mass of gas, enhancing the metallicity increases the surface density of planetesimals, increasing the chances for planet migration; (ii) if migration is halted by planetesimals hitting the star, then the planetesimals can pollute the surface layers of the star.

With several planets migration becomes more complex; for example, in the solar system Uranus and Neptune migrate outwards rather than inwards, because Jupiter acts like an inner absorbing boundary similar to a nearby stellar surface (23). In such a system the migration instability is triggered at a lower surface density, since the outer planets effectively push the inner planet toward the star. The migration of a massive planet will reduce the surface density of planetesimals substantially; as a result it is unlikely that two massive planets can migrate to short-period orbits. It is also unlikely that any other planets in the migration path can survive; thus a short-period Jupiter-mass planet should have no sister planets with orbital radii less than a few $\mathrm{AU}$. 
Table 1. Properties of planets

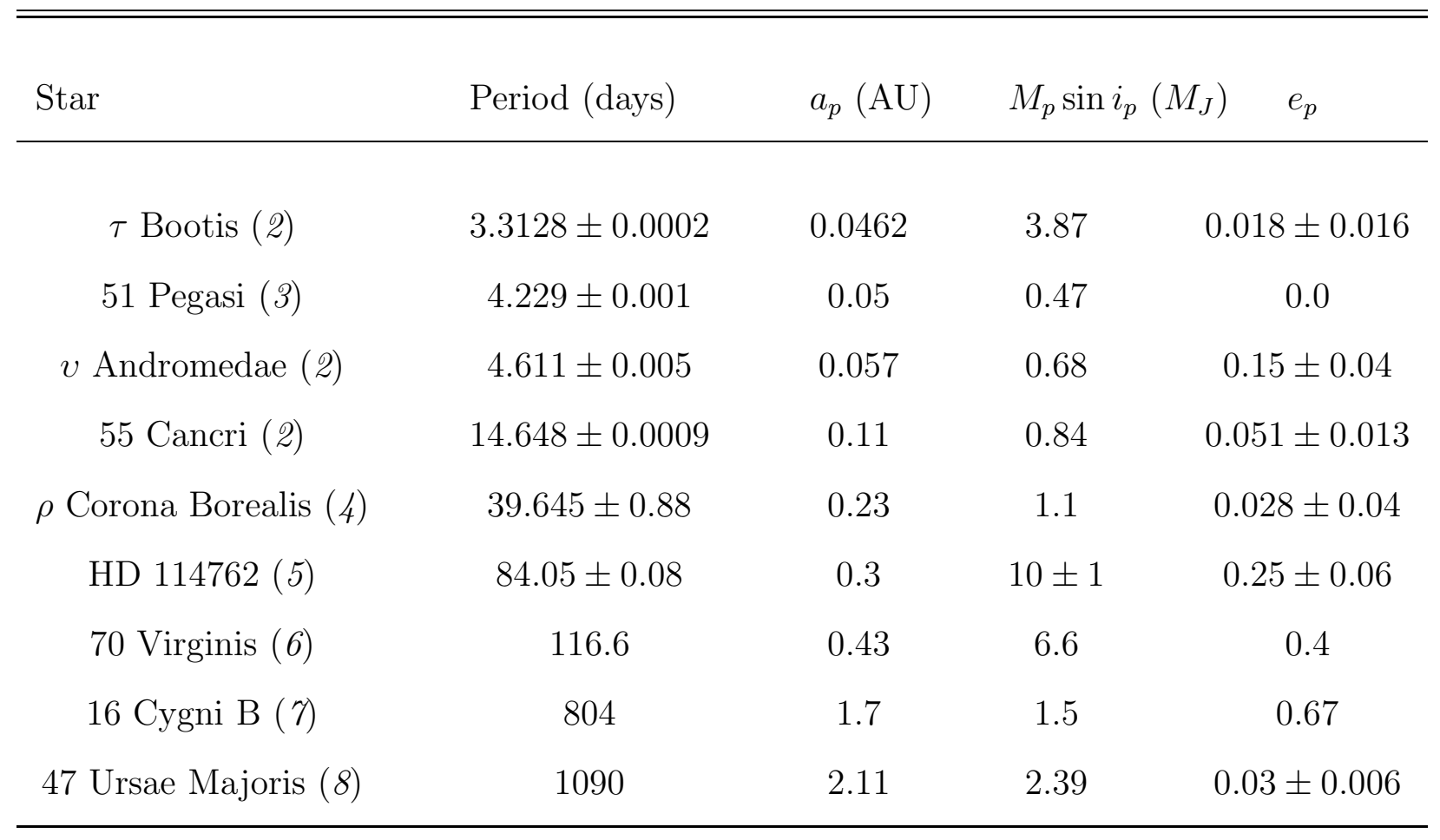




\section{REFERENCES}

1. J.J. Lissauer, Annu. Rev. Astron. Astrophys. 31, 129 (1993).

2. R.P. Butler, G.W. Marcy, E. Williams, H. Hauser, P. Shirts, Astrophys. J. 474, L115 (1997).

3. M. Mayor and D. Queloz, Nature 378, 355 (1995).

4. R.W. Noyes et al., Astrophys. J. 483, L111 (1997); see also erratum in Astrophys. J., 487, L195 (1997).

5. D.W. Latham, R.P. Stefanik, T. Mazeh, M. Mayor, G. Burki, Nature 339, 38 (1989).

6. G.W. Marcy and R.P. Butler, Astrophys. J. 464, L147 (1996).

7. W.D. Cochran, A.P. Hatzes, R.P. Butler, G.W. Marcy, Astrophys. J. 483, 457 (1997).

8. R.P. Butler, G.W. Marcy, Astrophys. J. 464, L153 (1996). See also the extra-solar planets catalog, http://www.obspm.fr:80/departement/darc/planets/catalog.html.

9. D. Gray, Nature 385, 795 (1996); G. Marcy et al., Astrophys. J. 481, 926 (1997).

10. D.N.C. Lin, P. Bodenheimer, D.C. Richardson, Nature 380, 606 (1996).

11. P. Goldreich, S. Tremaine, Astrophys. J. 241, 425 (1980).

12. F.A. Rasio and E.B. Ford, Science 274, 954 (1996).

13. S.J. Weidenschilling and F. Marzari, Nature 384, 619 (1996).

14. M. Holman and N. Murray, Astron. J. 112, 1278 (1996); N. Murray and M. Holman, Astron. J. 114, 1246 (1997); The presence of a massive planetesimal disk can change both the location and the amount of overlap relative to a Keplerian potential. The 
change in location will not affect the dynamics, and our calculations show that the change in the amount of overlap is generally small.

15. For resonances with $q \leq 2, e_{m}$ is replaced by $e$, but this expression must be used with caution when the resonances are strong, because the dynamics are more complicated; see $(14)$.

16. Somewhat longer times apply in other low-order mean-motion resonances. Similar results apply if the planet orbits inside the planetesimal.

17. If the planetary eccentricity $e_{p}$ is non-zero, there is a random walk in $J$, but this is much slower (by a factor of order $e_{p}$ ) than the random walk in $E$ or $L$.

18. H.F. Levison and M. Duncan, Icarus 108, 18 (1994). The lifetimes of Centaurs are similar; see L. Dones, H.F. Levison and M. Duncan, in Completing the Inventory of the Solar System, eds. T.W. Rettig and J.M. Hahn (San Francisco: Astronomical Society of the Pacific), 233 (1996).

19. Equation (2) holds for planets the mass and size of Jupiter with orbital periods larger than about 50 days.

20. B.V. Chirikov, Chaotic Dynamics in Hamiltonian Systems with Divided Phase Space, (Springer, Berlin, 1983); C.F.F. Karney, Physica D 8, 360, (1983); N. Murray, Physica D 52, 220 (1991).

21. H.F. Levison and M. Duncan, Icarus 127, 13 (1997); M.J. Duncan and H.F. Levison, Science 276, 1670 (1997).

22. By similar arguments, orbit-crossing planetesimals that collide with the star add energy to the planet; however, consumption by the star is much less common than ejection for planets at semimajor axes larger than a few stellar radii. 
23. J.A. Fernández and W.-H Ip, Icarus 58, 109 (1984).

24. J.-C. Liou and R. Malhotra, Science 275, 375 (1997).

25. The average radius $\alpha=\left(a / a_{p}\right)=(7 / 4)^{-2 / 3} \simeq 0.7$ if the inner $7: 4$ resonance is the only relevant one.

26. E.J. Öpik, Interplanetary Encounters (Elsevier Scientific, Amsterdam, 1976)

27. A. Toomre, Astrophys. J. 139, 1217 (1964); V.S. Safronov, Ann. d'Astrophys. 23, 979 (1960); J. Binney and S. Tremaine, Galactic Dynamics, (Princeton University Press, Princeton 1987).

28. M. Holman, J. Touma, S. Tremaine, Nature 386, 254 (1997); K.A. Innanen, J.Q. Zheng, S. Mikkola, M.J. Valtonen Astron. J. 113, 1915 (1997); T. Mazeh, Y. Krymolowski, G. Rosenfeld, Astrophys. J. 477, L103 (1997).

29. S.V.W. Beckwith, A.I. Sargent, R.S. Chini, R. Güsten, Astron. J. 99, 924 (1990).

30. A possible concern with the interpretation of these observations is that the gas surface density may imply gravitational instability if the disk extends out to 100 AU. See the references in $(27)$.

31. R.P. Butler, G.W. Marcy, E. Williams, H. Hauser, P. Shirts, Astrophys. J. 474, L115 (1997); S.L. Baliunas, G.W. Henry, R.A. Donahue, F.C. Fekel, W.H. Soon, Astrophys. J. 474, L119 (1977); G. Gonzalez, Mon. Not. R. Astron. Soc. 285, 403 (1997); G. Laughlin and F. C. Adams, astro-ph/9710113. Planet swallowing as a source of abundance anomalies in stellar atmospheres was first discussed by J.B. Alexander, The Observatory, 87, 238 (1967).

32. We acknowledge useful conversations with R. Malhotra and B. Paczyński. This research was supported by NSERC and by CIAR. 
Fig. 1. The evolution of the $i(t), a(t)$ and $e(t)$ of a planetesimal having a mass $10^{-2}$ that of Jupiter. The planetesimal was initially placed in the 7:4 resonance with $e=0.05$ and $i=0.05$. The perturbing planet had mass $\mu=10^{-3}$ and $e_{p}=0.05$. The planetesimal suffers its first close encounter with the planet after $3.5 \times 10^{5} \mathrm{yr}$, and is ejected after about $3.8 \times 10^{5} \mathrm{yr}$.

Fig. 2. The solid curves show the evolution of a 1 Jupiter-mass planet in a disk with planetesimal surface density $\Sigma(r)=\Sigma_{\odot}(1 \mathrm{AU} / r)^{3 / 2}$. We take initial elements as follows; the plane has $a_{p}=5.2 \mathrm{AU}$ and $e_{p}=0.048$, while the planetesimals have $a$ ranging from 0.03 to $4 \mathrm{AU}, e$ in the range $\left(e_{m}, e_{m}+0.2\right)$, and $i$ between 0 and 0.5 radians. The labelled curves correspond to the following values of $\Sigma_{\odot}$ in units of $\mathrm{g} \mathrm{cm}^{-2}$ : A) 8000, B) 2000, C) 1200, D) 600, and E) 40 (the nominal value for the minimum solar nebula). The corresponding gas surface densities in $\mathrm{g} \mathrm{cm}^{-2}$, assuming solar metallicity $Z=0.02$, are A) $\left.4 \times 10^{5}, \mathrm{~B}\right) 1 \times 10^{5}$, C) $6 \times 10^{4}$, D) $3 \times 10^{4}$, and E) $2 \times 10^{3}$. There is a critical value of $\Sigma_{\odot}$ at $\sim 200 \mathrm{~g} \mathrm{~cm}^{-2}$ (between D and E), below which there is very little movement. The solid circles indicate the positions of the various extrasolar planets (Table 1); the J indicates the location of Jupiter. The dotted line (labelled $\mathrm{A}^{*}$ ) corresponds to the case where we replaced the 2:1 resonance with the 3:1 resonance, in which the planetesimals are not strictly orbit-crossing unless one takes into account the finite Hill sphere radius. This indicates the approximate range of uncertainty in the final position in this idealized model. The time for the migration to occur is very model dependent; including more resonances will reduce the migration time. 


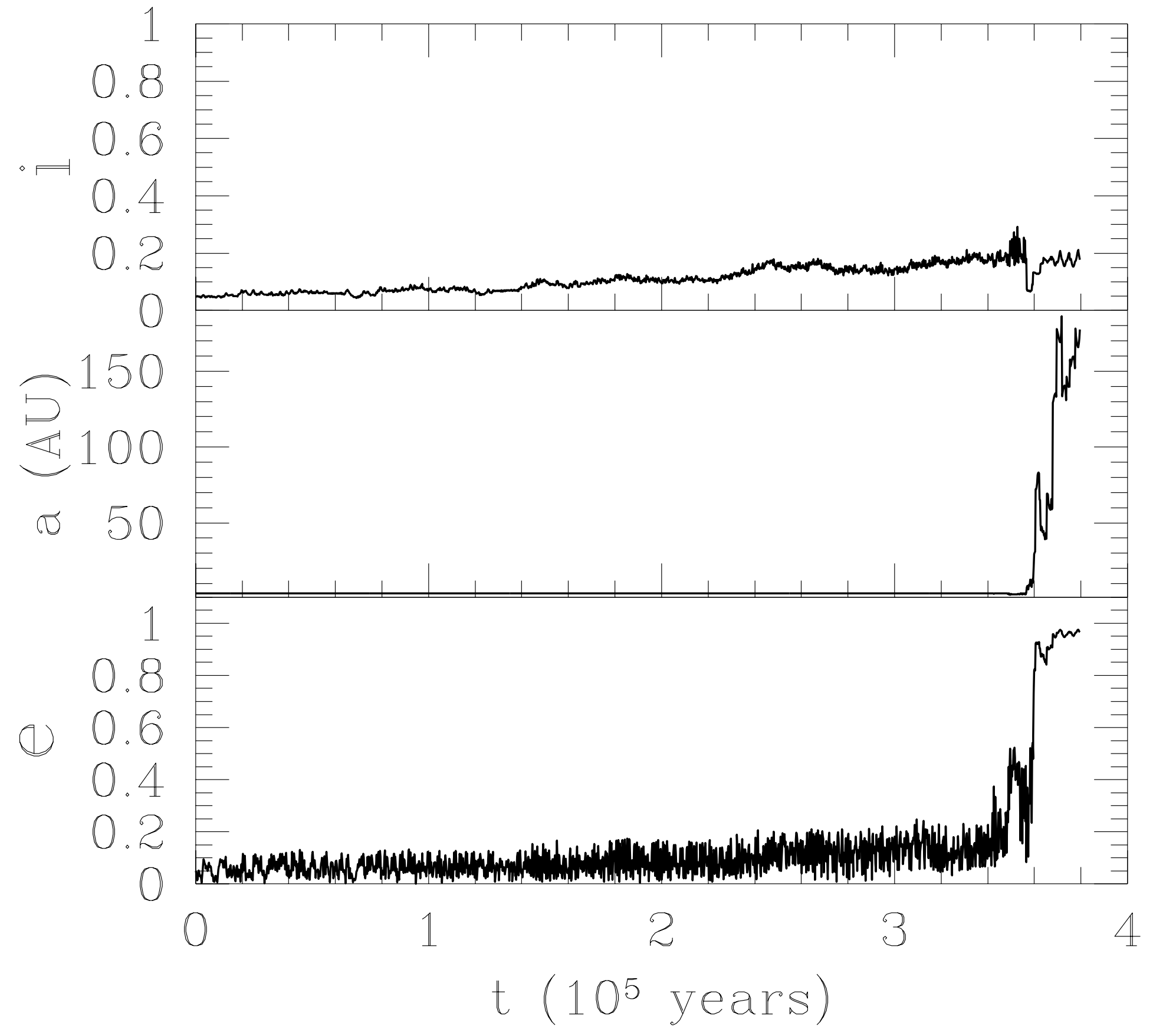




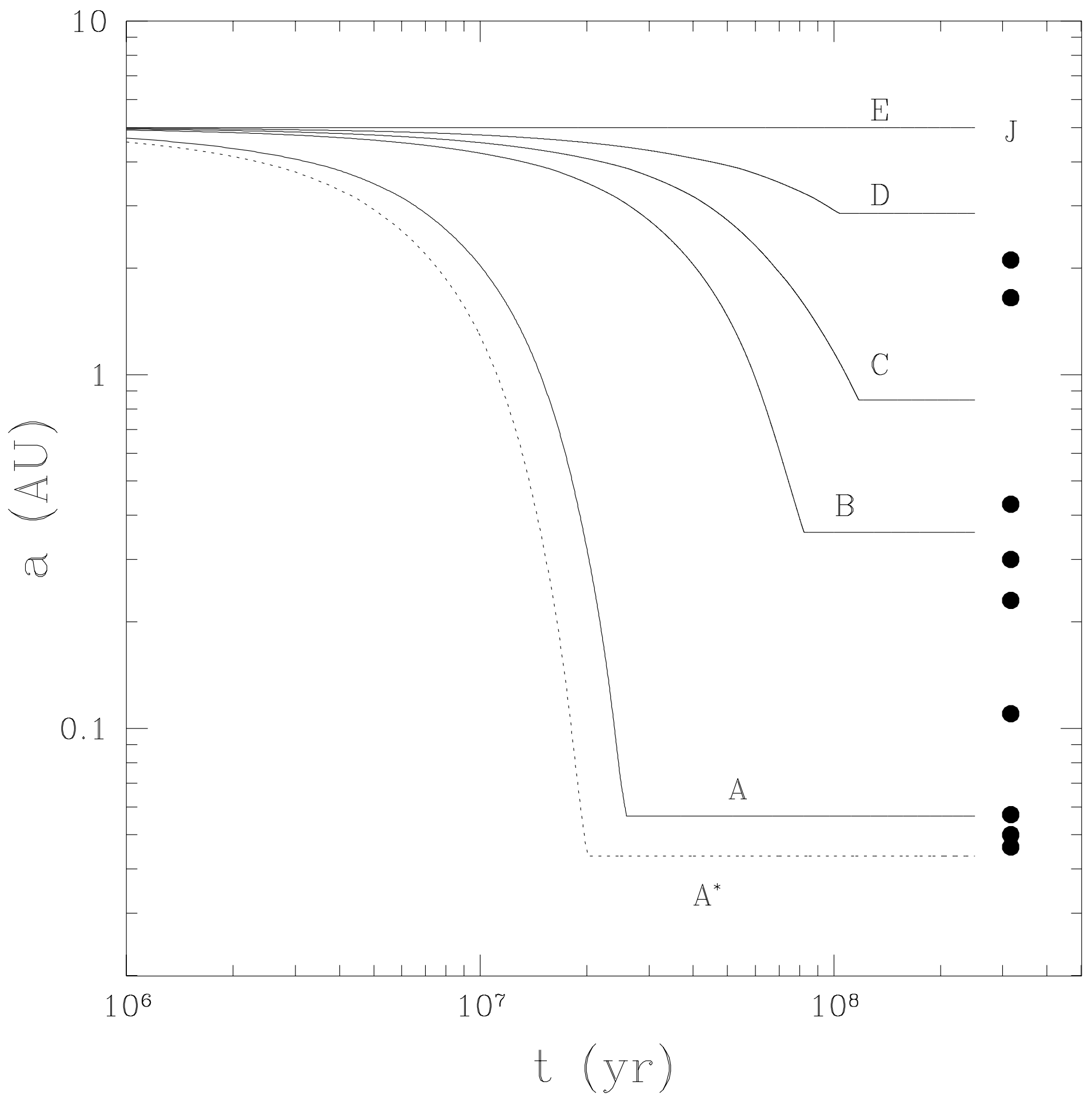

